\title{
A Brush Stroke Generation Using Magnetic Field Model for Painterly Rendering
}

\author{
Lee Soo Yeon, Yong Han Soon, and Yoon Kyung Hyun \\ 221, HukSeok-Dong, DongJak-Gu, Seoul, Korea, 156-756 \\ Computer Graphics Lab, CS\&E, Chung-Ang University \\ \{henim, hansoon, khyoon\} @cglab.cse.cau.ac.kr \\ http://cglab.cse.cau.ac.kr
}

\begin{abstract}
In this paper, we propose a new method for generating stroke which is the core of creating an image with a hand-painted appearance from a photograph. Magnetic Field Model is used in generating strokes and it is possible to create unique and natural brush strokes. In order to determine the direction of a stroke, a Magnetic Force Direction Map is made in the form of various circular and emissive shapes based on this model. We present two methods for generating stroke according to this direction map. We are able to create idiomatic and characteristic brush styles of a real painter using these methods.
\end{abstract}

\section{Introduction}

There are a lot of existing techniques to create a painted version of a digital photograph using computer. Figure 1 is a painting of Van Gogh which consists of brush strokes. Brush stroke gives a painting characteristic into the image. Therefore, many algorithms are developed to express strokes. Existing methods generated strokes using both straight lines and curved lines. So far, the algorithms[1,2,3] proposed for creating brush strokes using image gradients. But these methods have disadvantage that the direction of the strokes may appear artificial and cannot overcome the goal of creating strokes that express the character of a painter. Cha[4] created strokes with the consistent directionality by the region. This algorithm also has the same consistent directionality by each segmented area and does not express the various forms of the strokes. Therefore, it becomes difficult to express the brush strokes of real paintings using the image gradients only.

We suggest a magnetic field model for creating idiomatic strokes that can be found on paintings. The magnetic field model is created by the physical force between the magnetic objects, and the Magnetic Force Direction Map(MFDM) used to determine the direction of the stroke is acquired from the model. There are two different approaches to create strokes with specific styles using this map.

A vector field is created using the magnetic field model to create strokes freely with various styles. And then the MFDM is constructed to be applied on the direction of the stroke with it. We introduce two different approaches to create strokes using it. 


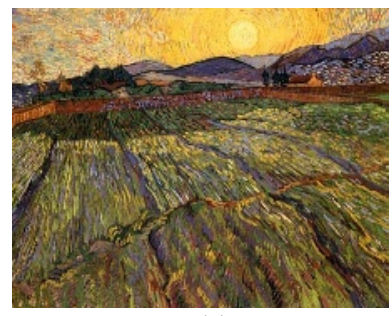

(a)

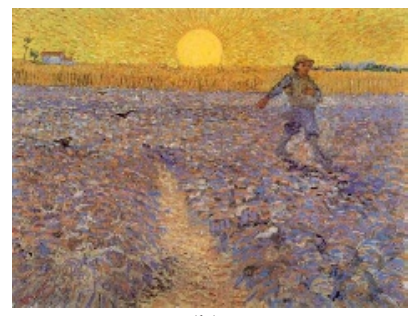

(b)

Fig. 1. Vicent van Gogh Oil on canvas, (a) wheat Rising with Sum, (b) Sower with Setting Sun, strokes around the sun are assumed the form of circular and emissive

The first approach is an automatic rendering method by the size of the brush. And the process is in the following. First, we acquire the gradient from the input image, and we reestablish it using MFDM which follows the magnetic field model. The reestablished direction draws a cubic B-Spline curve to create the stroke. In the second approach, the user adds strokes created by the MFDM on to the intermediate result image. The intermediate result image used for this process can be any image that was rendered by the existing algorithm. With the two approaches mentioned above, we can express the various types of strokes created by a human artist.

\section{Magnetic Field Model}

\subsection{Shape of Magnetic Field}

Magnetic force is a force that occurs between two magnetic objects and the space that is affected by it is called a magnetic field. A magnetic line of force is a virtual line used to indicate the magnetic force and the direction of the magnetic field is determined by the direction of the tangent line following the law of Ampere [5]. The magnetic force $(B)$ at the location that is perpendicularly away by a distance $r$ which is proportional to the force at the center of the magnetic field $(I)$ and is disproportional to the distance from the conducting wire $(r)$. It can be expressed by the following equation (1).

$$
B=k \frac{I}{r}
$$

A circular negative electric charge or positive electric charge grazing magnetic field is constructed around conducting wire where the current flows. The shape of the vector field also varies according to the number of conducting wire. Therefore, various shapes of vector fields can be created based on the magnetic field theory and the MFDM can be structured by this vector field to decide the direction of the stroke. 


\subsection{Vector Field Generation}

The location of the conducting wire is set up as the seed point and the currency is used as the input for creating the vector filed of the magnetic field model.

\section{Circular and Emissive}

To create a circular vector field, the current at the seed point $(I)$ and the conducting wire are used as the input. The distance affected by the current is calculated first and the directions of the tangent line for the magnetic field model are acquired to form the vector field. The direction of the magnetic field is acquired by using the fact that the tangent line of the circle is perpendicular to the half-circle crossing the point of contact and the characteristics of the vector. The direction and force of the user input currency become the parameter for creating the various number of vector field according to the number of seed points.

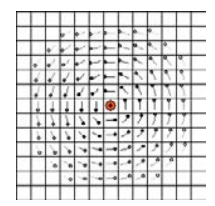

(a)

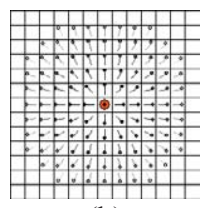

(b)

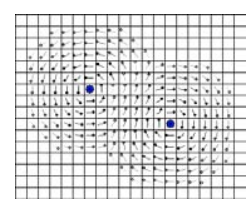

(c)

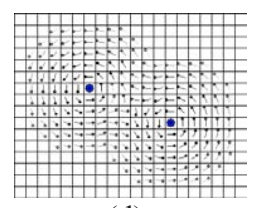

(d)

Fig. 2. Vector field. (a) Circular, (b) Emissive, (c),(d) User defined shape vector Field

Emissive vectors have either a positive electric charge or negative electric charge magnetic field as its model. The vector from a random point within the magnetic field to the input seed point can easily be acquired. (b) from Figure 2 shows the emissive vector fields and the magnetic field becomes larger as the currency becomes higher.

\section{User defined shape}

The conducting wire where the currency flows is used as the model, and the currency value is used as the seed point. All the vectors created by each currency from a random location $P$ are obtained for calculating the vector to a new direction. The number of seed points, direction and force of the currency are acquired through user input for creating various vector fields. (c) and (d) from Figure 2 each show vector fields created by setting two seed points with different direction.

The acquired vector has size and direction. The directions follow the magnetic field model, and the size at each location is express by the force of the magnetic field. The force of the magnetic field is reduced as the distance from the seed point increases until it is not affected at all at one point. The force of the magnetic field at a random point within the magnetic field is assigned as a gray-level value and is calculated as the ratio of the magnetic force at its current location against the maximum magnetic force. 


\subsection{Magnetic Force Direction Map}

The vector field created above becomes a main part of the magnetic force direction map. The MFDM is the same size as the input image, and it is consists of vector fields created by the magnetic force. Also, the user designates the area where the circular or emissive shape is applied. The created MFDM should go through a filtering stage for removing unnecessary images. Figure 3-(a) shows the result after a circular magnetic force direction map has been applied to the input image. Since there is no need for circular shaped strokes in drawing a mountain, the vectors are removed during the filtering stage. The removed area is replaced with gradients acquired from the input image and may be modified by the user.

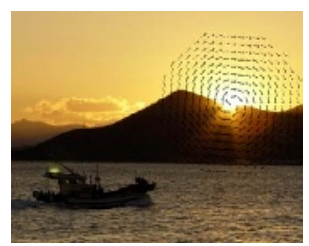

(a)

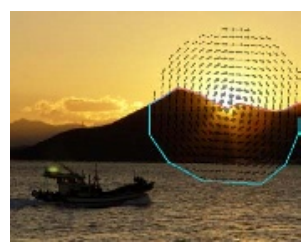

(b)

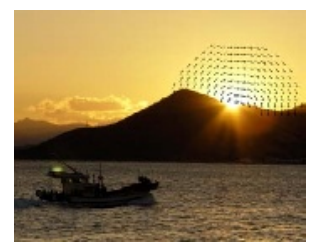

(c)

Fig. 3. (a) Result image which is apply MFDM to the source image, (b) select the region that will be removed unnecessary vector, (c) result image of removing the vector for the selected region

\section{Stroke Rendering}

We introduce two algorithms for creating strokes. They are common in that they use the magnetic force direction map through referencing the magnetic field model. The first method applies an automatic method for rendering the whole canvas through converting the gradient of the selected region to the direction of the magnetic force direction map. The second method applies rendering by adding an extra stroke that follows the magnetic force direction map onto the original image. The two methods differ in the rendering method used.

\subsection{Curved Brush Stroke}

The user selects the area for either a circular or emissive stroke from the input image. A magnetic force direction map is created for the selected area and is applied to the input image to re-establish the direction of the gradient through the magnetic force direction map. The brush stroke is created using the cubic B-Spline curve. The direction of the stroke follows the magnetic force direction map and the other areas refer the direction of the gradient. The parameters of the stroke such as the starting point, color, length, and depth are applied by changing the curved line brush stroke creation algorithm[3]. 


\subsection{Magnetic Field Based Stroke}

The strokes around the sun as shown in Figure 9 (a), a work of Van Gogh, have different directions and colors from that used to draw the sky. The two new algorithms in this study, produce various shapes of strokes by adjusting the force of the magnetic field through changing the parameters. The result image using this method can reflect the original image, compared to other methods using image gradients.

Figure 4 (a) shows the process for creating a circular brush stroke. The starting point is set at a location with a distance of $r$ from the seed point and creates strokes by locating a control point every $l$ to the direction of the vector. The distance between the strokes $\theta$ is set randomly, and the condition for finishing the strokes from the circle is set as a pre-defined angle, which in this case was set to $30^{\circ}$.

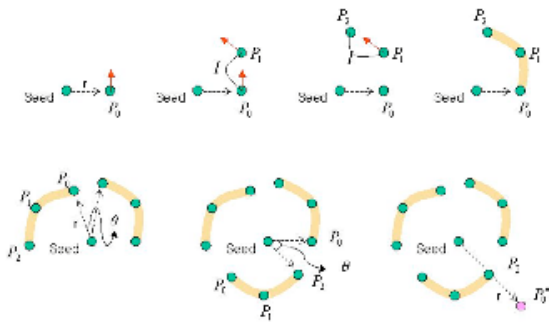

(a)

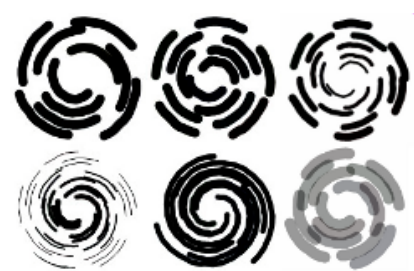

(b)

Fig. 4. (a) The process of creating circular stroke, (b) circular stroke

Figure 5 (a) shows the process for creating the emissive brush stroke, and the resulting brush stroke is shown in (b). The starting point is randomly selected from the created magnetic field, and the length and the thickness is determined by the force of the magnetic field. The strokes are created bi-directionally from the starting point and ends when it contacts the seed point.

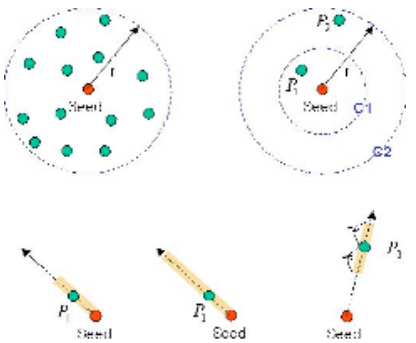

(a)

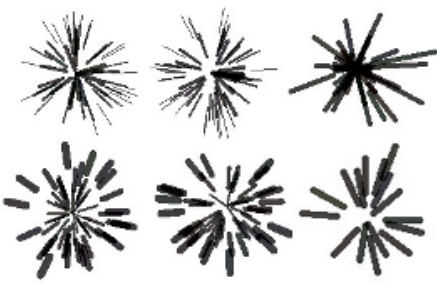

(b)

Fig. 5. (a) The process of creating emissive stroke, (b) emissive stroke 


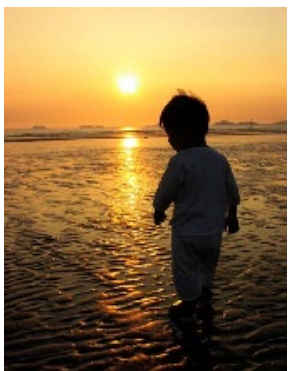

(a)

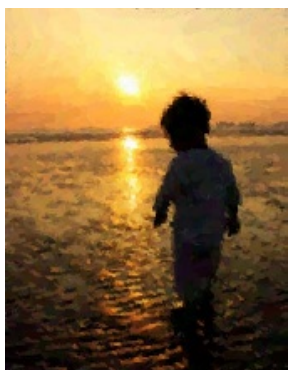

(b)

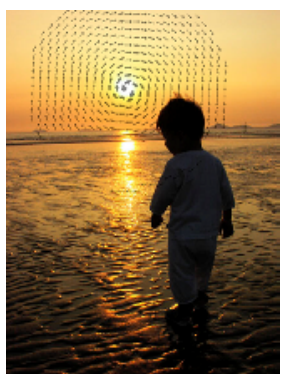

(c)

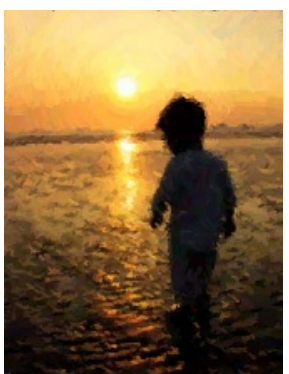

(d)

Fig. 6. The process of applying circular MFDM to the source image. (b) the result of using gradient, (d) is the result image when the direction map of (c) was applied

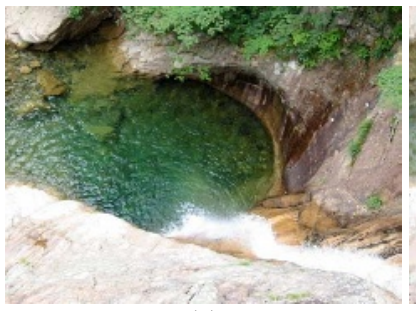

(a)

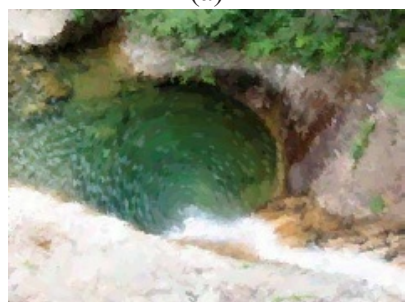

(d)

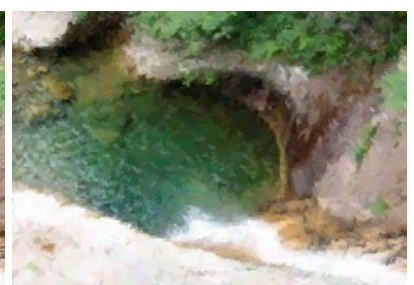

(b)

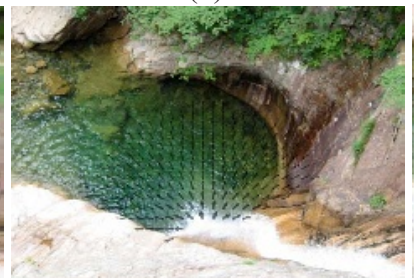

(e)

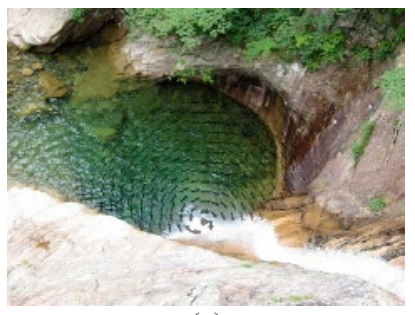

(c)

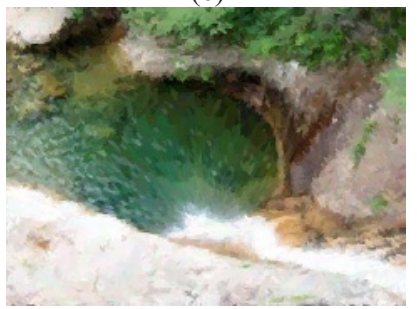

(f)

Fig. 7. Result images of applying circular and emissive MFDM to the source image. (a) input image, (b) result image using gradient, (c),(e) are the results of applying circular and emissive MFDM to the source image, and the result rendered images are (d),(f)

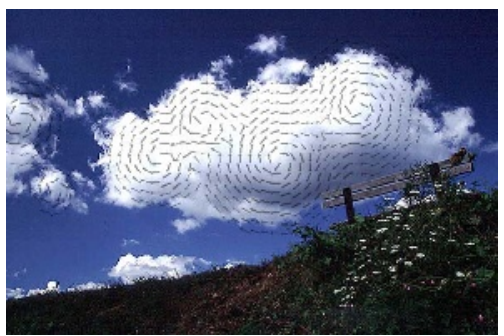

(a)

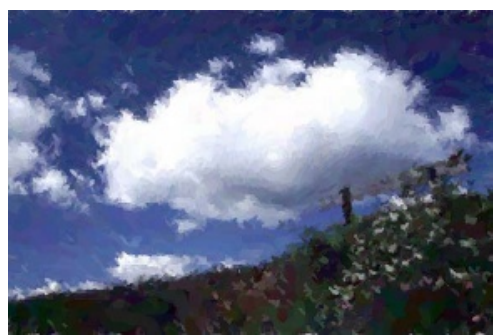

(b)

Fig. 8. Result which is rendered by using user defined MFDM. The result of (a) is each (b) 


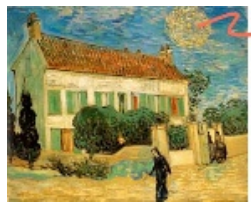

(a) Whiter House, Gogh

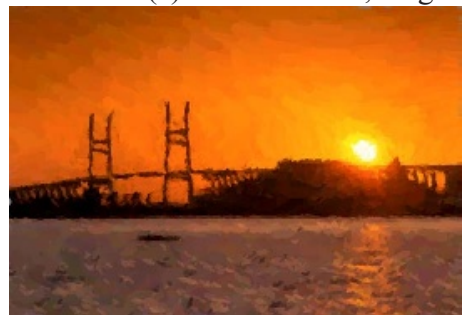

(c)

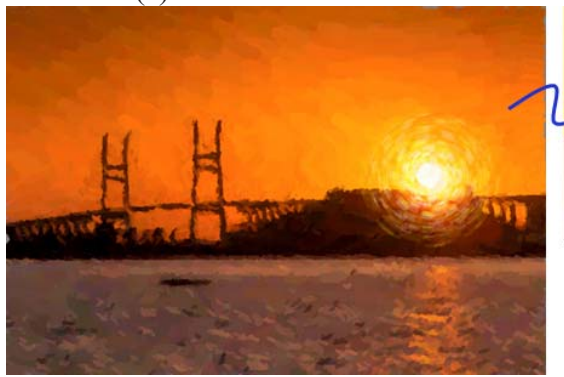

(e)

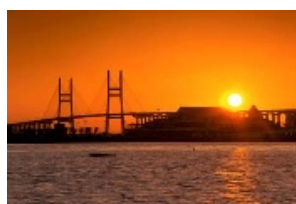

(b) 입력 영상

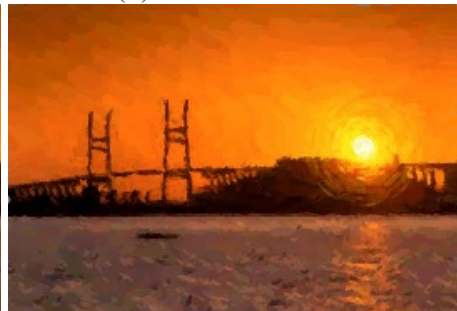

(d)

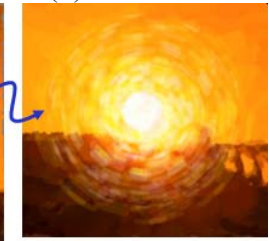

Fig. 9. (a) The painting of Gogh, (c) intermediate result image, (e) is created of circular stroke through $(d)$
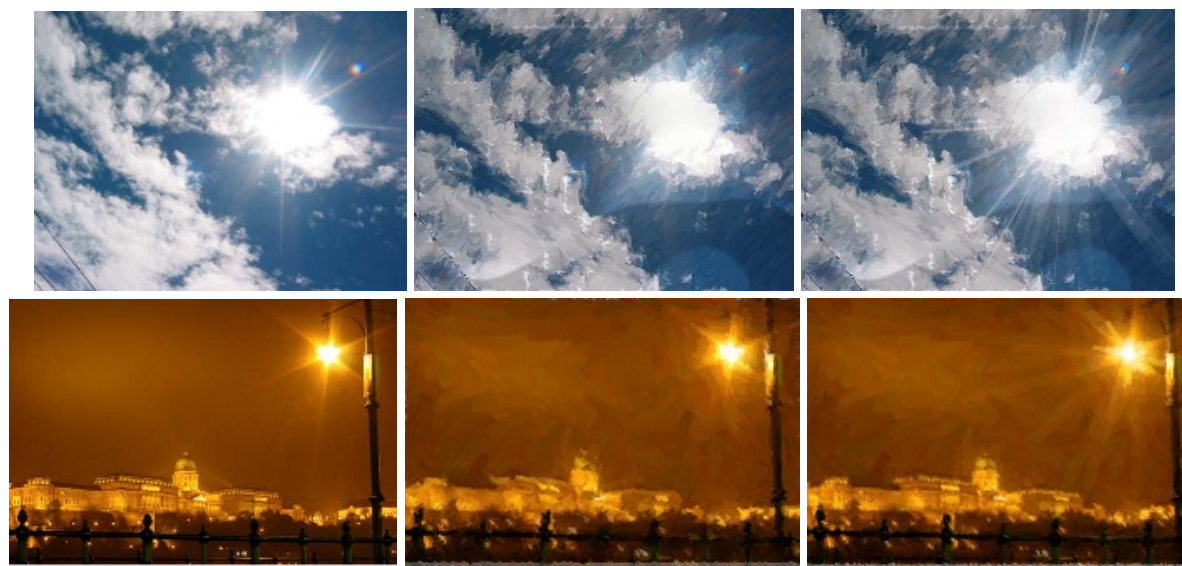

(b)

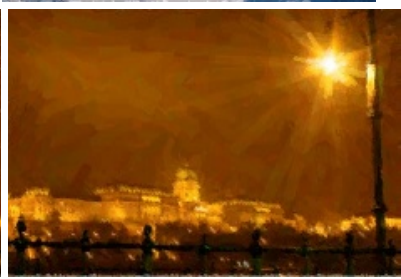

(c)

Fig. 10. (a) Rows are input image, (c) rows are the result image of applying emissive stroke to the result image which is rendered by using gradient as (b) rows 


\section{Result and Future Work}

In this paper, a Magnetic Field Model is proposed to create strokes to make a painterly rendering image. As the result, we can represent the circular and emissive stroke and furthermore, we can illustrate idiomatic brush style of artists with various MFDM.

There is an advantage that the effect of a source image is reflected in the result image as in the figure 9 and 10 . However, several troublesome tests have to be gone through in order to achieve these good result images.

It is complicated but possible to produce 2-dimension animations: the scene for the intensity of the light is gradually stronger or weaker and for the movement of the clouds, by strokes only from one source image. The images which are including streetlights or stars and sunset or sunrise are proper with the source image. And the blending phenomenon which is observed in actual paintings and illustration of quality and texture need to be simulated for the further study.

This work was partially supported by the National Research Laboratory program of the Korean Ministry of Science and Technology (No. 2000-N-NL-01-C-285) and by the ITRI of Chung-Ang University in 2004

\section{References}

1. Paul Haeberli, Paint by Numbers : Abstract image representations, SIGGRAPH90 Proceeding (1990) 207-214

2. Peter Litwinowicz, Processing Images and Video for an Impressionist Effect, In SIGGRAPH 97 (1997) 407-414

3. Aaron Hertzmann, Painterly rendering with curved brush strokes of multiple sizes, SIGGRAPH98 Proceeding (1998)

4. Jeong Seob Cha, A Directional Stroke Generation Algorithm for Painterly Rendering, LNCS2669 (2003) 326-335

5. Zoya Popovic, Branko D. Popovic, Introductory Electromagnetics, Prentice Hall (2000) 